\title{
电弧放电法制备石墨烯及其在导电油墨中的应用
}

\author{
杨 洁 $^{1,2}$, 潘争辉 ${ }^{2}$, 盛雷梅 ${ }^{1}$, 安 康 $^{1}$, 赵新洛 ${ }^{1}$
}

(1. 上海大学 低维炭材料与器件物理研究所, 上海 200444; 2. 中国科学院 苏州纳米技术与纳米仿生研究所, 苏 州 215123)

摘 要: 导电油墨是印刷电子技术中使用的关键电子材料, 而导电填料作为导电油墨的主要成分要求其化学性能稳 定且电导率高。其中，基于石墨烯的导电油墨因为其优异的电学性质受到广泛关注。本研究采用直流电弧放电法制 备的石墨烯作为导电填料，并通过扫描电子显微镜、透射电子显微镜、拉曼光谱等手段对制备的石墨烯进行了表征。 结果表明: 直流电弧放电法制备的石墨烯为 2 10 层、尺寸在 100 200 nm 范围且纯度高、结晶性好。在此基础上，研 究了涂层厚度、热处理温度以及弯曲角度等对石墨烯导电油墨导电性能的影响。研究发现, 石墨烯导电油墨电阻率 与涂层厚度、热处理温度成反比，且随着厚度、温度的增加石墨烯导电油墨的电阻率逐渐降低。并且样品在柔性基 底上经过不同角度的弯曲折叠后电阻率没有明显变化。当厚度为 $170 \mu \mathrm{m}$ 的样品经过 $360^{\circ} \mathrm{C}(30 \mathrm{~min})$ 热处理后, 石 墨烯导电油墨的电阻率仅为 $0.003 \Omega \cdot \mathrm{cm}$ 。上述结果表明，电弧法制备的石墨烯导电油墨有望成为未来印制电子领域 的关键材料。

关 键 词: 石墨烯; 电弧放电; 导电油墨

中图分类号: TQ174 文献标识码: A

\section{Graphene Nanosheets Prepared by Arc Discharge Method and Their Application in Conductive Inkjet}

\author{
YANG Jie $^{1,2}$, PAN Zheng-Hui ${ }^{2}$, SHENG Lei-Mei $^{1}$, AN Kang ${ }^{1}$, ZHAO Xin-Luo ${ }^{1}$
}

(1. Institute of Low-dimensional Carbons and Device Physics, Shanghai University, Shanghai 200444, China; 2. Suzhou Institute of Nano-Tech and Nano-Bionics, Chinese Academy of Sciences, Suzhou 215123, China)

\begin{abstract}
Conductive ink is one of the dominant electronic materials for printed electronics technology. As the main component in conductive inks, conductive fillers are required to have favorable electronic properties and good chemical stability. Up to date, graphene-based conductive ink has attracted enormous research interest mainly due to its remarkable electrical properties. In present study, the graphene sheets as the conductive fillers were fabricated via a direct current arc discharge evaporation method. Furthermore, the as-prepared graphene sheets were characterized by scanning electron microscope (SEM), transmission electron microscope (TEM) and Raman spectra analysis. The results demonstrated that the graphene sheets consisting of 2-10 layers with sheet size ranging from $100 \mathrm{~nm}$ to $200 \mathrm{~nm}$ had high purity and high crystallinity. Besides, the relationships between conductive properties and coating thicknesses, annealing temperatures and bending angles of conductive ink, were also carried out. It was found that the resistivity of conductive ink was inversely proportional to its thickness and annealing temperature, i.e. with gradual increase in thickness and temperature, the resistivity was decreased. And the resistivity of the conductive ink on the flexible sub-
\end{abstract}


strate remained stable under different folding angles. Specially, the conductive ink with a thickness of $170 \mu \mathrm{m}$ achieved the specific resistivity of $0.003 \Omega \cdot \mathrm{cm}$ after being annealed at $360^{\circ} \mathrm{C}$ for $30 \mathrm{~min}$. The results indicate that arc-discharge graphene sheets are promising alternative for next generation of printed electronics.

Key words: graphene sheets; arc discharge; conductive ink

导电油墨是一种由导电填料、连接剂、溶剂和 助剂组成的导电性复合材料, 属于功能性特种油墨, 通常印刷于导电承印物上, 具有传导电流、排除静 电荷积累的作用 ${ }^{[1-3]}$ 。按照导电填料种类, 大体上可 将导电油墨分为贵金属系填充、非贵金属系填充及 无机碳类填充导电油墨。贵金属系(金、银)导电油 墨 ${ }^{[1]}$ 、非贵金属系(铜)导电油墨 ${ }^{[4]}$ 、碳系导电油墨都 已成功应用于水晶和薄膜太阳能光电板、FRID、逻 辑和记忆电路、触屏显示器、电子传感器以及智能 包装等商业化领域中 ${ }^{[5-7]}$ 。但是，由金、银以及其它 贵金属制成的导电油墨由于价格昂贵, 应用范围受 到一定限制 ${ }^{[3]}$ 。相比之下, 碳系导电油墨具有成本低 和性能稳定等优点, 且不需要太多印后处理步骤。 因此, 碳系导电油墨的开发和性能研究引起了科研 人员的广泛关注 ${ }^{[8-10]}$ 。

常用碳系导电油墨的填充材料主要有石墨、碳 粉、碳纳米管、碳纤维和石墨烯等, 其中基于石墨、 碳粉的导电油墨尽管价格低廉, 但是其电导率低, 易因固化后收缩过大导致油墨层开裂，使其应用受 阻 ${ }^{[11-12]}$ 。以碳纳米管和石墨烯为代表的碳基纳米材料 在导电油墨中的应用越来越受关注 ${ }^{[8,13]}$ 。与碳纳米管 和碳纤维相比, 石墨烯具有更大的比表面积 $\left(2630 \mathrm{~m}^{2} / \mathrm{g}\right)$, 更为优异的电子传导性能 $\left(2 \times 10^{5} \mathrm{~cm}^{2} /(\mathrm{V} \cdot \mathrm{s})\right)^{[14]}$, 且在 $10 \sim 100 \mathrm{~K}$ 范围内并未表现出温度的依赖性 ${ }^{[15-16]}$, 因 此受到更多的关注。

石墨烯常见的制备方法有微机械剥离法、化学 剥离法、化学气相沉积法以及电弧放电法等。其中, 微机械剥离法和化学气相沉积法制备的石墨烯虽然 质量高, 但是产量低, 难以实现石墨烯的规模化制

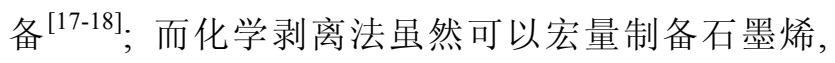
但是氧化、超声以及后续还原往往会造成碳原子的 缺失, 使得石墨烯含有较多缺陷, 导电性变差 ${ }^{[19-20]}$; 由电弧放电法制备的石墨烯, 不仅具有高达 $600^{\circ} \mathrm{C}$ 以上的氧化温度、高的结晶性和纯度, 而且可以在 实验室中实现石墨烯样品的克量级制备, 甚至有望 放大为公斤级甚至吨级的工业规模, 目前已逐渐成 为制备高质量石墨烯的主要方法。

为了实现高电导率导电油墨的规模化制备, 满 足其在印制电子器件中的需求, 本工作采用电弧放
电法制备的石墨烯为导电填料, 希望能够解决传统 方法中导电填料成本高、电导率低等问题, 从而为 未来石墨烯导电油墨在电子器件和储能器件中的应 用提供一定基础。

\section{1 实验方法}

\section{1 石墨烯的制备}

在自制的水冷不锈钢真空腔内, 利用直流电弧 放电法制备石墨烯 ${ }^{[1]}$ 。压强为 $53200 \mathrm{~Pa}$, 氢气和氮 气混合气体体积比为 $1: 1$, 两直径为 $10 \mathrm{~mm}$ 的高纯 石墨棒做为放电电极。整个合成过程中，电极间距 通过步进马达始终控制在 $2 \mathrm{~mm}$ 左右, 放电电流为 $120 \mathrm{~A}$, 放电过程约持续 $30 \mathrm{~min}$ 。放电过程中, 纯石 墨棒的阳极被蒸发, 石墨烯沉积在不锈钢真空室的 内壁上, 每次可以产生克量级的高纯度粉末状石墨 烯片。

\section{2 石墨烯导电油墨的制备}

首先将电弧法制得的石墨烯和乙基纤维素( EC, 阿法埃莎化学试剂有限公司)的混合物(质量比 $5: 1$ ) 加入到体积比为 $1: 2.5$ 的松油醇(AR, 阿拉丁化学试 剂有限公司)和乙醇(GR, 国药集团化学试剂有限公 司)的混合溶液中; 然后用磁力搅拌器(S21-2, 上海 司乐仪器有限公司)搅拌 $30 \mathrm{~min}$, 得到黑色的悬浮液; 最后用超声波清洗器(KQ3200DE, 昆山市超声仪器 有限公司)分散 $24 \mathrm{~h}$ (超声功率 $100 \mathrm{~W}$ ), 得到石墨 烯导电油墨。为了研究涂层厚度、热处理温度以及 弯曲角度等对石墨烯导电油墨导电性能的影响, 先 利用涂布机(MSK-AFA-III Automatic Thinck Film Coater 合肥科晶技术有限公司)分别把合成的石墨 烯导电油墨涂覆在载玻片和 PET 膜上, 然后在真空 干燥箱(DZF 系列真空干燥箱, 上海右一仪器有限 公司) 中 $60{ }^{\circ} \mathrm{C}$ 干燥 $24 \mathrm{~h}$, 最后在石英管式炉 (OTF-1200X, 合肥科晶技术有限公司)中不同温度 下热处理。

\section{3 表征}

利用扫描电子显微镜(Hatichi SF-4800 型)观察 石墨烯的表面形貌; 利用透射电子显微镜(Tecnai F-30FEG 型)观察石墨烯的微观形貌; 利用拉曼光谱 
测试仪(Lab RAM HR 型)对石墨烯进行结构表征。

利用多功能数字式四探针测试仪(ST-2258A 型, 苏州晶格电子有限公司)对石墨烯导电油墨进行电 阻率的测试。

\section{2 结果与讨论}

\section{1 石墨烯样品的形貌与结构}

图 1 是典型的电弧法制备的石墨烯样品的扫描 电子显微镜和透射电子显微镜照片。从图 1(a)中可 以看到电弧法制备的石墨烯呈花瓣状结构, 且这些 花瓣的尺寸主要在 100 200 nm 之间, 石墨烯样品 表面中几乎不存在其它杂质或者无定形碳, 说明电 弧法制备的石墨烯样品纯度高, 结晶性好。此外, 石 墨烯表面褶皱明显，表现出不同层次的透明度 ${ }^{[22]}$ 。 石墨烯的层数可以通过石墨烯褶皱边缘的高分辨透 射电子显微镜照片来判断。图 1(c e ) 是 5、8 和 9 层 石墨烯(分别对应图 b 中的 A、B 和 C 区域)的 HRTEM 照片, 说明电弧法制备的石墨烯的层数大 约为 $2 \sim 10$ 层。

拉曼光谱作为一种无破坏, 快速表征的手段, 常用来研究石墨烯的缺陷、均匀性和层数 ${ }^{[22]}$ 。图 2(a) 是电弧法制备的少层石墨烯典型的拉曼光谱图。石墨
烯的拉曼光谱呈现三个特征峰 D 峰、 $G$ 峰和 $2 D$ 峰: $\mathrm{D}$ 峰位于 $1352 \mathrm{~cm}^{-1}$, 由边缘、不规则 $\mathrm{C}\left(\mathrm{sp}^{3}\right.$ 键) 等石 墨的无序性诱导引起 ${ }^{[23]}$; $\mathrm{G}$ 峰位于 $1585 \mathrm{~cm}^{-1}$, 是由 石墨一阶拉曼的 $E_{2 g}$ 光学模引起的 ${ }^{[24]}$, 该峰强度较 高, 宽度较窄, $\mathrm{sp}^{2}$ 杂化占主导, 一般为单峰; $2 \mathrm{D}$ 峰

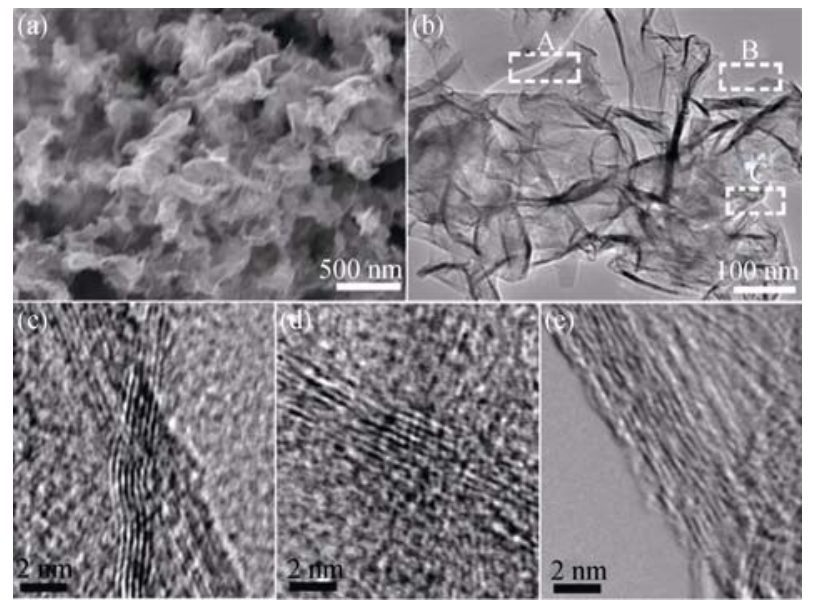

图 1 (a)电弧法制备石墨烯的扫描电子显微镜照片, (b)透射 电子显微镜照片和(c e e) 5、8 和 9 层石墨烯(分别对应 $\mathrm{b}$ 图中的 A、B 和 C 区域)的高分辨透射电子显微镜照片

Fig. 1 (a) SEM and (b) TEM images of arc-discharge graphene sheets prepared by arc discharge evaporation; (c-e) HRTEM images of graphene sheets with 5, 8 and 9 layers taken from region A, B and C in Fig. 1(b), respectively
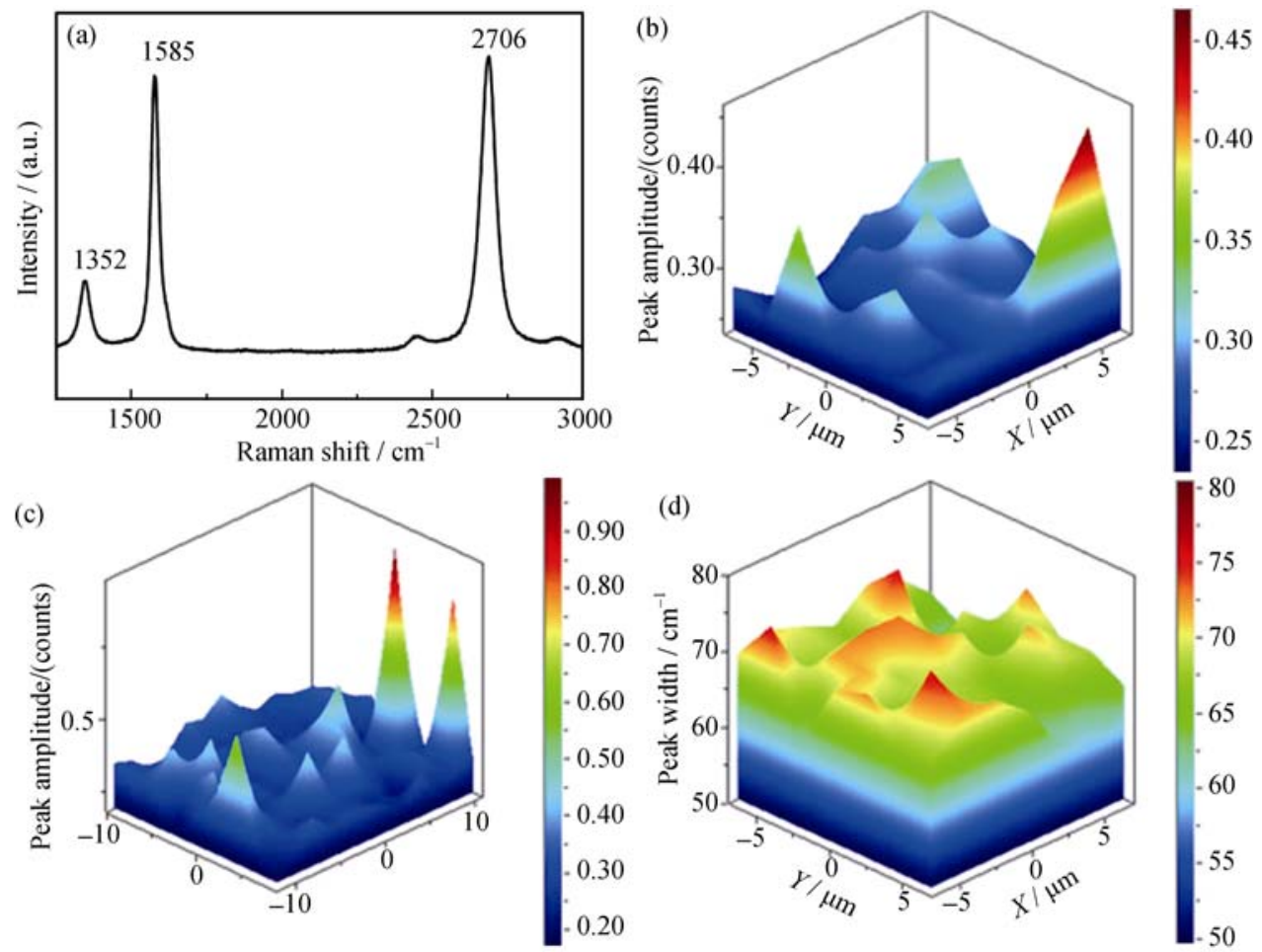

图 2 电弧法制备的少层石墨烯的拉曼光谱图

Fig. 2 Raman spectra of a few layer graphene sheets prepared by arc discharge evaporation (a) Typical Raman spectrum of graphene sheets; (b) D/G peak ratio mapping; (c) G/2D peak ratio mapping; (d) FWHM Raman mapping 
位于 $2706 \mathrm{~cm}^{-1}$, 由双光子在第一布里渊区中心的两 个互不等价的 $\mathrm{K}$ 点附近双共振拉曼激发引起 ${ }^{[25]}$ 。 $\mathrm{D}$ 峰与 $\mathrm{G}$ 峰的强度比值 $\left(I_{\mathrm{D}} / I_{\mathrm{G}}\right)$ 通常用来表示石墨材料 的缺陷程度, 且反比于石墨烯的面内尺寸 $L_{\mathrm{a}}$ 。根据 公式 $L_{\mathrm{a}}=2.4 \times 10^{-10} \cdot \lambda^{4} \cdot\left(I_{\mathrm{G}} / I_{\mathrm{D}}\right)^{[26]}, \lambda$ 为激光波长 $514 \mathrm{~nm}$, $I_{\mathrm{D}} / I_{\mathrm{G}}=0.17$, 可以得到 $L_{\mathrm{a}}=2.9 \mathrm{~nm}$ 。说明电弧法制备的 石墨烯片层之间存在一定的间隙, 不存在明显的堆 叠。图 2(b)是面积为 $50 \mu \mathrm{m} \times 50 \mu \mathrm{m}$ 的电弧法制备的 少层石墨烯 $I_{\mathrm{D}} / I_{\mathrm{G}}$ 的 mapping 图, 从图中可以看出 $I_{\mathrm{D}} / I_{\mathrm{G}}$ 的值在 $0.1 \sim 0.9$ 之间, 并且 $80 \%$ 的面积里 $I_{\mathrm{D}} / I_{\mathrm{G}}$ 在 0.3 以下, 证明电弧法制备的石墨烯不仅均匀性 好而且质量高。据报道, $2 \mathrm{D}$ 峰与 $\mathrm{G}$ 峰的强度比值 $\left(I_{2 \mathrm{D}} / I_{\mathrm{G}}\right)$ 和 $2 \mathrm{D}$ 峰的半宽高值 $(\mathrm{FWHM})$ 的数值与石墨 烯的层数有关 ${ }^{[18,22,27]}$, 可以用来估算石墨烯的层数。 $\mathrm{FWHM}<45 \mathrm{~cm}^{-1}$ 且 $I_{2 \mathrm{D}} / I_{\mathrm{G}}>1.4$ 时, 为单层石墨烯 ${ }^{[17]}$; FWHM 处于 $45 \sim 60 \mathrm{~cm}^{-1}$ 之间且 $I_{2 \mathrm{D}} / I_{\mathrm{G}}$ 在 $0.75 \sim 1.4$ 范 围内时, 为双层石墨烯; 而当 $F W H M>60 \mathrm{~cm}^{-1}$ 且 $I_{2 \mathrm{D}} / I_{\mathrm{G}}<0.75$ 时, 为少层石墨烯 ${ }^{[28-29]}$ 。图 2(c)和(d)分 别是面积为 $50 \mu \mathrm{m} \times 50 \mu \mathrm{m}$ 的电弧法制备的石墨烯的 $I_{2 \mathrm{D}} / I_{\mathrm{G}}$ 的 mapping 图和 $2 \mathrm{D}$ 峰的 FWHM 的 mapping 图, 可以看出, 电弧法制备的石墨烯样品为 2 10 层的 少层石墨烯, 这与 HRTEM 分析结果相符合。以上结 果表明, 电弧法制备的石墨烯纯度高, 且层数在 2 10 层之间, 适于用作导电填料制备石墨烯导电油墨。

\section{2 石墨烯导电油墨的拉曼光谱}

图 3 是不同涂层厚度及不同温度退火的石墨烯 导电油墨的拉曼光谱图, 从图中可以看出, 石墨烯 导电油墨的拉曼光谱同样有三个主要的特征峰, 分 别是位于 $1585 \mathrm{~cm}^{-1}$ 附近的 $\mathrm{G}$ 峰, 位于 $1364 \mathrm{~cm}^{-1}$ 附 近的 D 峰和位于 $2721 \mathrm{~cm}^{-1}$ 附近的 $2 \mathrm{D}$ 峰。在室温下, 随着样品厚度增加, $\mathrm{D}$ 峰的强度有微小减弱, 这是 因为随着涂层厚度的增加, 石墨烯导电油墨中的有 机基体对石墨烯的包覆作用导致拉曼信号减弱; 但
是不同涂层厚度样品拉曼峰的趋势保持不变说明电 弧法制备的石墨烯导电油墨十分均匀。图 3(b)显示, 厚度为 $170 \mu \mathrm{m}$ 的样品, 当退火温度升高到 $400^{\circ} \mathrm{C}$ 时, D 峰的强度基本上没有变化, 说明石墨烯导电油墨 的缺陷没有增加，证明电弧法制备的石墨烯导电油 墨可以承受 $400^{\circ} \mathrm{C}$ 的高温环境; 并且 $2 \mathrm{D}$ 峰与 $\mathrm{G}$ 峰的 强度比值、2D 峰的半高宽值都基本保持不变, 进一 步说明电弧法制备的导电油墨的化学性能稳定, 可 用于耐高温环境。

\section{3 不同条件下石墨烯导电油墨的电阻率变化}

图 4(a)是制备好的石墨烯导电油墨静置 $30 \mathrm{~d}$ 后 的照片，从图中可以看出电弧法制备的石墨烯导电 油墨均匀性良好, 且无明显的分层现象 ${ }^{[30]}$ 。图 4(b) 给出了室温下、弯曲角度为 $0^{\circ}$ 时, 石墨烯导电油墨 电阻率和厚度的关系, 电阻率随厚度的增加而降低, 因为油墨在干燥固化之前, 导电填料处于均匀分散 状态, 填料间接触不稳定, 无导电性。当油墨干燥或 者固化后, 溶剂的挥发和连接料的固化使油墨体积 收缩, 填料间形成导电网络结构, 呈现导电性。且随 着涂层厚度的增加, 该导电网络中的载流子的浓度 增加, 导电通路增多。因此随着厚度的增加, 石墨烯 导电油墨电阻率减小, 导电性加强。当石墨烯导电 油墨厚度为 $170 \mu \mathrm{m}$ 时, 电阻率仅为 $0.072 \Omega \cdot \mathrm{cm}$, 证 明电弧法制备的石墨烯导电性良好。石墨烯导电油墨 中的乙基纤维素会随着温度的升高而炭化(如图 4(c) 插图), 使得油墨的导电性明显提高。因此, 为了研究 石墨烯导电油墨的电阻率与退火温度的关系, 将石墨 烯导电油墨均匀地涂覆在载玻片上(厚度为 $170 \mu \mathrm{m}$, 弯曲角度为 $0^{\circ}$ ), 探究了不同温度热处理后电阻率的 变化情况。如图 4(c) 所示, 选取五个热处理温度(分别 为 $23^{\circ} \mathrm{C} 、 160^{\circ} \mathrm{C} 、 240^{\circ} \mathrm{C} 、 360^{\circ} \mathrm{C}$ 和 $400^{\circ} \mathrm{C}$ ), 在氮气气 氛下, 分别对厚度为 $170 \mu \mathrm{m}$ 的样品退火 $30 \mathrm{~min}$ 。结 果显示, 随着热处理温度的升高, 样品的电阻率
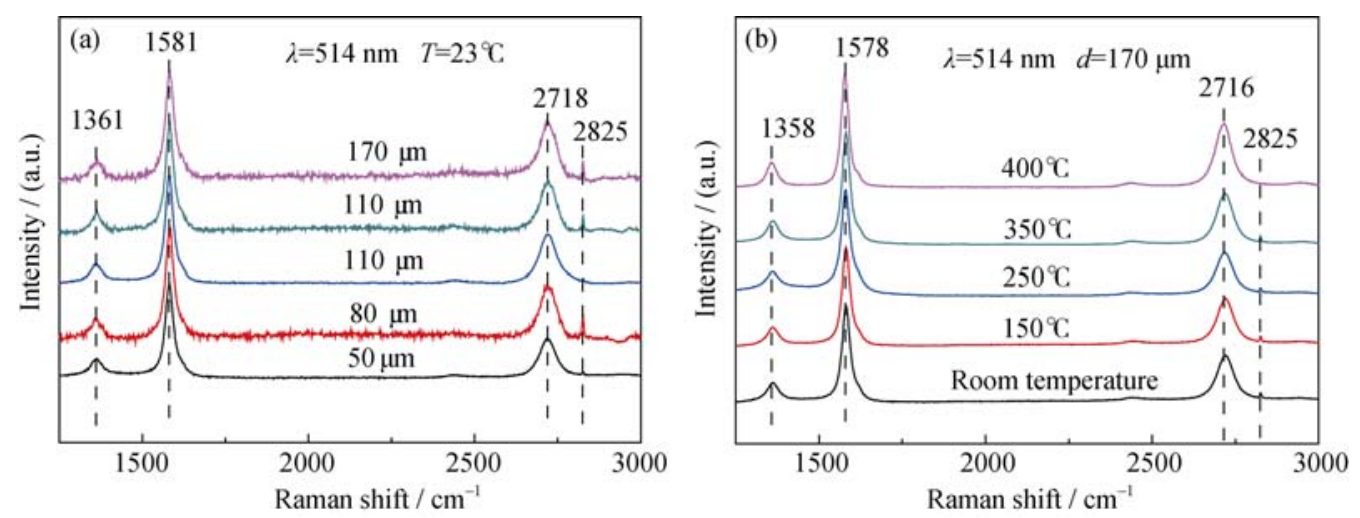

图 3 (a)不同厚度; (b)不同温度退火的石墨烯油墨的拉曼光谱图

Fig. 3 Raman spectra of graphene conductive ink with (a) different thicknesses and (b) being annealed at different temperatures 

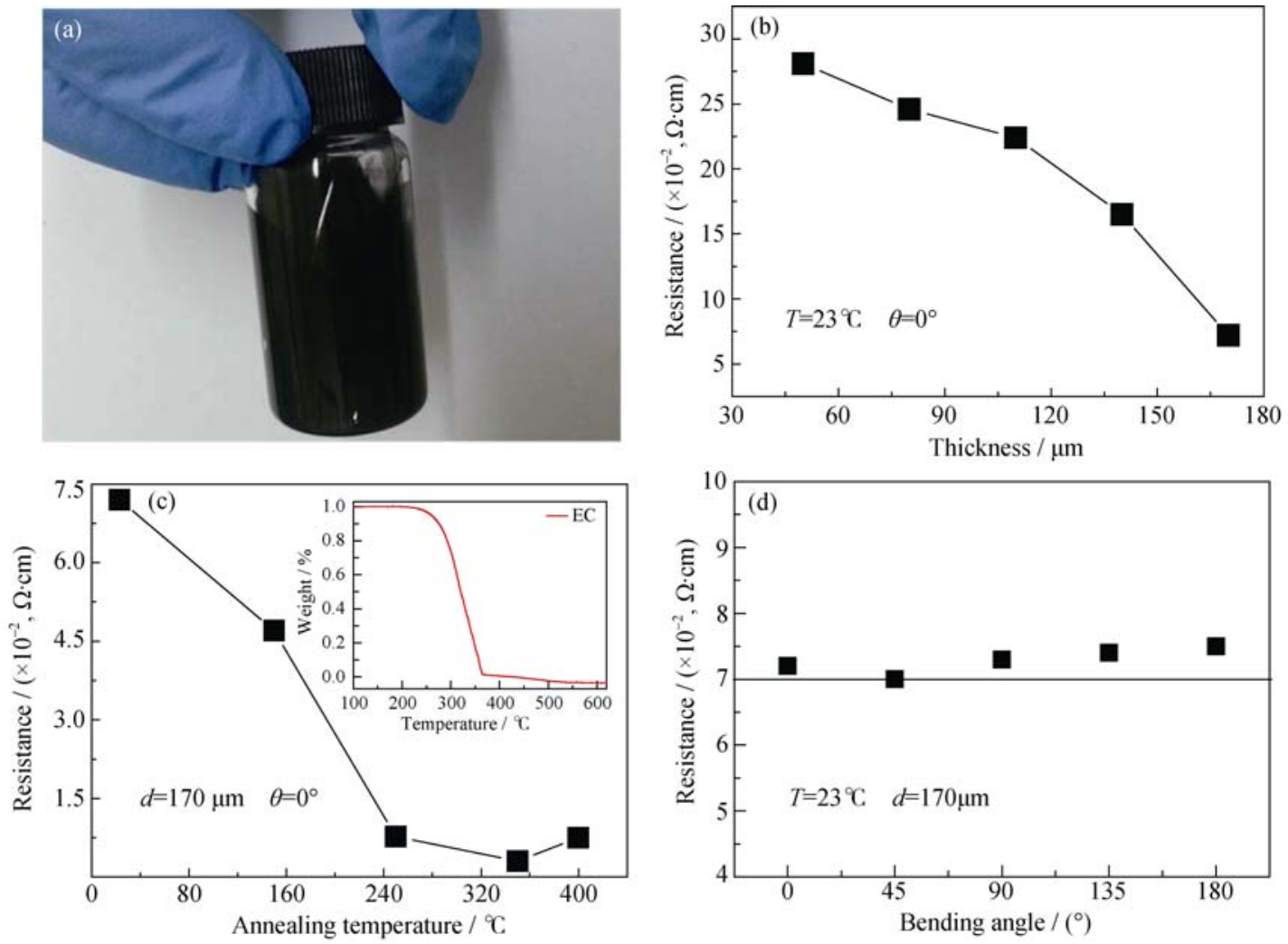

图 4 静置 $30 \mathrm{~d}$ 后石墨烯导电油墨分散液的照片(a), 石墨烯基导电油墨的电阻率与涂层厚度的关系(b); 电阻率与退火温度的关 系(插图为乙基纤维素的热重分析图) (c); 电阻率与弯曲角度的关系(d)

Fig. 4 (a) Picture of graphene conductive ink after being laid for 30 d, Relationship between resistivity and thickness(b), annealing temperature(c) (The insert showing thermogravimetric analysis (TGA) of ethyl cellulose (EC)), and bending angle (d) of graphene conductive ink

降低, 导电性变好, 因为热处理温度接近 $250^{\circ} \mathrm{C}$ 时 (如图 4(c)插图)乙基纤维素开始分解为芳香类产物, 它们能与石墨烯形成 $\pi-\pi$ 键堆垛, 从而提供更有效 的导电通道。当热处理温度为 $360^{\circ} \mathrm{C}(30 \mathrm{~min})$ 时, 电 阻率达到最小值 $0.003 \Omega \cdot \mathrm{cm}$; 但是, 当热处理温度 升高到 $400^{\circ} \mathrm{C}$ 时, 电阻率又略微提高, 这是由于残 留的乙基纤维素完全蒸发热解而引起的(如图 4(c) 插图)。同时为了评估电弧法制备的石墨烯导电油墨 的机械性能, 我们把导电油墨涂布在 PET 膜上(室 温下, 厚度为 $170 \mu \mathrm{m})$ 。图 4(d)显示了石墨烯导电油 墨电阻率和弯曲角度的关系, 可以明显看出, 样品 经过不同角度弯曲后, 电阻率没有明显的变化, 基 本维持在 $0.075 \Omega \cdot \mathrm{cm}$ 左右, 说明电弧法制备的石 墨烯导电油墨具有良好的柔韧性，可以应用到柔 性、可折叠电子器件中, 大大拓宽了其应用领域 ${ }^{[31]}$ 。

\section{3 结论}

1) 利用电弧放电法制备的石墨烯具有产量高、 纯度高、结晶性好等优点。

2) 利用电弧法制备的石墨烯作为导电填料合
成石墨烯导电油墨，制备的导电油墨分散性好，无 明显分层。

3) 涂覆在载玻片上厚度为 $170 \mu \mathrm{m}$ 石墨烯导电 油墨, 经过 $360^{\circ} \mathrm{C}$ 热处理后, 电阻率仅为 $0.003 \Omega \cdot \mathrm{cm}$, 说明电弧法制备的石墨烯导电油墨不仅化学性能稳 定、能耐高温, 且导电性能优异。

4) 经过不同角度弯曲后，电阻率没有明显的 变化, 基本维持在 $0.075 \Omega \cdot \mathrm{cm}$ 左右，说明电弧法制 备的石墨烯导电油墨具有良好的柔韧性，可以应 用到柔性、可折叠电子器件中, 大大拓宽了其应用 领域。

\section{参考文献:}

[1] JAHN S F, BLAUDECK T, BAUMANN R R, et al. Inkjet printing of conductive silver patterns by using the first aqueous particle-free MOD ink without additional stabilizing ligands. Chemistry of Materials, 2010, 22(10): 3067-3071.

[2] WU J T, HSU S L C, TSAI M H, et al. Direct inkjet printing of silver nitrate/poly(N-vinyl-2-pyrrolidone) inks to fabricate silver conductive lines. The Journal of Physical Chemistry C, 2010, 114(10): 4659-4662. 
[3] LEE H H, CHOU K S, HUANG K C. Inkjet printing of nanosized silver colloids. Nanotechnology, 2005, 16(10): 2436-2441.

[4] RATHMELL A R, WILEY B J. The synthesis and coating of long, thin copper nanowires to make flexible, Transparent Conducting films on plastic substrates. Advanced Materials, 2011, 23(41): 4798-4803.

[5] HABAS S E, PLATT H A S, VAN HEST M F A M, et al. Low-Cost inorganic solar cells: from ink to printed device. Chemical Reviews, 2010, 110(11): 6571-6594.

[6] $\mathrm{KOH} \mathrm{C} \mathrm{G,} \mathrm{TAN} \mathrm{W,} \mathrm{ZHAO} \mathrm{M} \mathrm{Q,} \mathrm{et} \mathrm{al.} \mathrm{Integrating} \mathrm{polymerase}$ chain reaction, valving, and electrophoresis in a plastic device for bacterial detection. Analytical Chemistry, 2003, 75(17): 4591-4598.

[7] BAEG K J, CAIRONI M, NOH Y Y. Toward printed integrated circuits based on unipolar or ambipolar polymer semiconductors. Advanced Materials, 2013, 25(31): 4210-4244.

[8] GEIM A K, NOVOSELOV K S. The rise of graphene. Nature Materials, 2007, 6(3): 183-191.

[9] HUANG L, HUANG Y, LIANG J, et al. Graphene-based conducting inks for direct inkjet printing of flexible conductive patterns and their applications in electric circuits and chemical sensors. Nano Research, 2011, 4(7): 675-684.

[10] SECOR E B, PRABHUMIRASHI P L, PUNTAMBEKAR K, et al. Inkjet printing of high conductivity, flexible graphene patterns. Journal of Physical Chemistry Letters, 2013, 4(8): 1347-1351.

[11] SIVARAMAKRISHNAN S, CHIA P J, YEO Y C, et al. Controlled insulator-to-metal transformation in printable polymer composites with nanometal clusters. Nature Materials, 2007, 6(2): 149-155.

[12] CHEN H, M LlER M B, GILMORE K J, et al. Mechanically strong, electrically conductive, and biocompatible graphene paper. Advanced Materials, 2008, 20(18): 3557-3561.

[13] NOVOSELOV K S, GEIM A K, MOROZOV S V, et al. Electric field effect in atomically thin carbon films. Science, 2004, 306(5696): 666-669.

[14] STOLLER M D, PARK S, ZHU Y, et al. Graphene-based ultracapacitors. Nano Letters, 2008, 8(10): 3498-3502.

[15] MIĆIĆ O I, SMITH B B, NOZIK A J. Core-shell quantum dots of lattice-matched $\mathrm{ZnCdSe}_{2}$ shells on InP cores: experiment and Theory. The Journal of Physical Chemistry B, 2000, 104(51): 12149-12156.

[16] RAVINDRAN S, CHAUDHARY S, COLBURN B, et al. Covalent coupling of quantum dots to multiwalled carbon nanotubes for electronic device applications. Nano Letters, 2003, 3(4): 447-453.

[17] LI X, CAI W, AN J, et al. Large-area synthesis of high-quality and uniform graphene films on copper foils. Science, 2009, 324(5932): $1312-1314$.
[18] REINA A, JIA X, HO J, et al. Large area, few-layer graphene films on arbitrary substrates by chemical vapor deposition. Nano Letters, 2009, 9(1): 30-35.

[19] HERNANDEZ Y, NICOLOSI V, LOTYA M, et al. High-yield production of graphene by liquid-phase exfoliation of graphite. Nature Nanotechnology, 2008, 3(9): 563-568.

[20] LOTYA M, HERNANDEZ Y, KING P J, et al. Liquid phase production of graphene by exfoliation of graphite in surfactant/water solutions. Journal of the American Chemical Society, 2009, 131(10): 3611-3620.

[21] CHEN Y, ZHAO H, SHENG L, et al. Mass-production of highlycrystalline few-layer graphene sheets by arc discharge in various $\mathrm{H}_{2}$-inert gas mixtures. Chemical Physics Letters, 2012, 538: $72-76$.

[22] FERRARI A C, MEYER J C, SCARDACI V, et al. Raman spectrum of graphene and graphene layers. Physical Review Letters, 2006, 97(18): 187401-1-4.

[23] TAN P, HU C, DONG J, et al. Polarization properties, high-order Raman spectra, and frequency asymmetry between Stokes and anti-Stokes scattering of Raman modes in a graphite whisker. Physical Review B, 2001, 64(21): 214301-1-12.

[24] TAN P H, HAN W P, ZHAO W J, et al. The shear mode of multilayer graphene. Nature Materials, 2012, 11(4): 294-300.

[25] ZHAO W J, TAN P H, ZHANG J, et al. Charge transfer and optical phonon mixing in few-layer graphene chemically doped with sulfuric acid. Physical Review B, 2010, 82(24): 245423-1-9.

[26] PIMENTA M A, DRESSELHAUS G, DRESSELHAUS M S, et al. Studying disorder in graphite-based systems by Raman spectroscopy. Physical Chemistry Chemical Physics, 2007, 9(11): 1276-1291.

[27] GRAF D, MOLITOR F, ENSSLIN K, et al. Spatially resolved Raman spectroscopy of single- and few-layer graphene. Nano Letters, 2007, 7(2): 238-242.

[28] YAN K, PENG H, ZHOU Y, et al. Formation of bilayer bernal graphene: layer-by-layer epitaxy via chemical vapor deposition. Nano Letters, 2011, 11(3): 1106-1110.

[29] CHEN S, CAI W, PINER R D, et al. Synthesis and characterization of large-area graphene and graphite films on commercial $\mathrm{Cu}-\mathrm{Ni}$ alloy foils. Nano Letters, 2011, 11(9): 3519-3525.

[30] SINGH M, HAVERINEN H M, DHAGAT P, et al. Inkjet printing-process and its applications. Advanced Materials, 2010, 22(6): $673-685$.

[31] SECOR E B, PRABHUMIRASHI P L, PUNTAMBEKAR K, et al. Inkjet printing of high conductivity, flexible graphene patterns. The Journal of Physical Chemistry Letters, 2013, 4(8): 1347-1351. 